\title{
The impact of COVID-19 pandemic on global mental health: From the general public to healthcare workers
}

\author{
Roger C $\underline{\text { Ho }},{ }^{1,2_{F R C P S y c h}}$, Bach X Tran, ${ }^{3,4_{P h D}}$, Roger S McIntyre, ${ }^{5,6,7,8_{F R C P C}}$
}

The COVID-19 pandemic began in late 2019 and was declared a pandemic by the World Health Organization (WHO) on 11 March 2020. To decrease the spread of the virus and demand on the healthcare system, governments globally executed multiple public health measures including lockdown, ${ }^{1}$ social distancing, ${ }^{2}$ significant closure of the economy, as well as curbing domestic and international travel.

More than a year after the foregoing unprecedented interventions were implemented across countries around the world, tremendous uncertainty remains. This includes the availability of vaccinations and therapeutics, containment of the virus due to the emergence of variants, the return of the employment sector to pre-2020 levels, as well as the availability of unrestricted travel and social activities. Approximately two-thirds of households reported income loss due to the impact of the pandemic, which differentially affected specific sociodemographic groups (e.g. women, ethnic and racial minorities, individuals in unskilled labour positions). ${ }^{2}$ During the lockdown, a surfeit of factors were identified that are associated with an increased level of depression, stress and anxiety, including but not limited to being female, single, younger age (i.e. $<30$ years), separated, widowed, impact of rumours, behavioural changes, losing jobs, and being in contact with potential COVID-19 patients. ${ }^{2-4}$ The potential hazardous effects of COVID-19 on mental health have become an international public health priority. ${ }^{5}$ Mental health research, which aims to identify risk and resiliency factors for mental health and mental disorders during this unprecedented crisis, is most likely to be achieved by international collaboration representing the disparate and global effect of COVID-19.
For middle-income countries in Asia, the risk factors for adverse mental health during the pandemic identified were age younger than 30 years, high education background, single status, and potential discrimination by people from other countries for spreading the COVID-19 virus. ${ }^{6}$ In contrast, mental health protective factors include family support from cohabiting family members, employment, confidence in doctors, high perceived likelihood of survival, and spending less time on health information. ${ }^{6}$ The foregoing finding of a link between less time on health information and mental health protection is in accordance with a separate observation that excess time on social media was associated with increased anxiety, stress and depression during COVID-19. ${ }^{7}$

The aforementioned studies also identified that being in physical contact with potential COVID-19 patients was a risk factor for poorer overall mental health. Compared with the general population, healthcare workers are expected to have higher exposure rates to patients who suffered from COVID-19 infection, and long working hours during the pandemic could lead to burnout. ${ }^{8}$ A recent study involving healthcare workers from Asian countries found that non-medically trained personnel, the presence of physical symptoms, and prior medical conditions were all independent predictors for an increased likelihood of greater severity ratings on psychiatric scales. ${ }^{9}$ Among healthcare workers from India, Indonesia, Malaysia, Singapore and Vietnam, Singaporean healthcare workers had a low prevalence of depression and anxiety. ${ }^{9}$ Possible explanations could be due to the vast experience of Singaporean healthcare workers in handling previous pandemics such as the outbreak of severe acute respiratory syndrome (SARS) in 2003 .

\footnotetext{
Department of Psychological Medicine, Yong Loo Lin School of Medicine, National University of Singapore, Singapore

${ }^{2}$ Institute of Health Innovation and Technology (iHealthtech), National University of Singapore, Singapore

${ }^{3}$ Institute for Preventive Medicine and Public Health, Hanoi Medical University, Hanoi, Vietnam

${ }^{4}$ Bloomberg School of Public Health, Johns Hopkins University, Baltimore, MD, United States of America

${ }^{5}$ Mood Disorders Psychopharmacology Unit, University Health Network, Toronto, ON, Canada

${ }^{6}$ Department of Psychiatry, University of Toronto, Toronto, ON, Canada

7 Department of Pharmacology, University of Toronto, Toronto, ON, Canada

${ }^{8}$ Brain and Cognition Discovery Foundation, Toronto, ON, Canada

Correspondence: A/Prof Roger C Ho, Department of Psychological Medicine, NUS Yong Loo Lin School of Medicine, 1E Kent Ridge Road, NUHS Tower Block Level 9, Singapore 119228.

Email: pcmrhcm@nus.edu.sg
} 
Previous research on the mental health of Singaporean healthcare workers during the COVID-19 pandemic was conducted on healthcare workers who provided medical services for adults and elderly. ${ }^{10}$ The latest study by Kirk et al. reported in this issue of the Annals assessed the psychological impact of the pandemic on paediatric healthcare workers in the middle of Phase I of the "circuit breaker" - the stayat-home order and cordon sanitaire implemented as a preventive measure by the Singapore government. ${ }^{11}$ This study found a relatively high prevalence of depression, anxiety and stress in paediatric healthcare workers at $39.1 \%, 47.7 \%$ and $24.7 \%$, respectively. The foregoing prevalence rates are higher than the overall prevalence rates reported in other studies evaluating Asian healthcare workers (depression 4.5\%, anxiety $5.2 \%$, and stress $1 \%),{ }^{9}$ as well as a previous study on Singapore's healthcare workers (depression $8.9 \%$, anxiety $14.5 \%$, and stress $6.6 \%) .{ }^{10}$ However, the findings by Kirk et al. are within the range of psychological response rates observed among healthcare workers during previous epidemics ${ }^{12}$ and current COVID-19 pandemic, ${ }^{13}$ based on recent reviews of a large number of extant studies. Kirk et al. had identified female gender, lack of choice on work scope/ environment, insufficient physical activity, and stigma as key risk factors for poorer mental health outcomes in paediatric healthcare workers.

One interesting finding came out of the study. In contrast to the hypothesis that long working hours are associated with burnout and negative mental health, there was an inverse association between work hours per week and anxiety levels in paediatric healthcare workers. Kirk et al. proposed that those workers who volunteered to work longer were mentally more prepared for looking after COVID-19 patients. ${ }^{11}$ The other possible explanation is that the long working hours made paediatric healthcare workers become desensitised to fear and anxiety associated with the pandemic.

Finally, this study found that organisational measures such as open, bidirectional communication with senior management, support from the hospital and family, and adequate personal protective equipment were associated with lower stress levels in healthcare workers. During the pandemic, online psychotherapy such as Internet-based cognitive behaviour therapy can reduce negative thoughts and behaviours by offering a safer alternative to face-to-face counselling. ${ }^{14}$ Towards the aim of safeguarding the mental health of healthcare workers in Singapore, it is imperative to identify and modify risk factors, as well as embolden resiliency factors among this critical workforce.

\section{REFERENCES}

1. Le HT, Lai AJX, Sun J, et al. Anxiety and Depression Among People Under the Nationwide Partial Lockdown in Vietnam. Front Public Health 2020;8:589359.

2. Tran BX, Nguyen HT, Le HT, et al. Impact of COVID-19 on Economic Well-Being and Quality of Life of the Vietnamese During the National Social Distancing. Front Psychol 2020; 11:565153.

3. Long VJE, Liu JCJ. Behavioural changes during the COVID-19 pandemic: Results of a nationwide survey in Singapore. Ann Acad Med Singap 2021;50:222-31.

4. Long VJE, Koh WS, Saw YE, et al. Vulnerability to rumours during the COVID-19 pandemic in Singapore. Ann Acad Med Singap 2021;50:232-40.

5. Xiong J, Lipsitz O, Nasri F, et al. Impact of COVID-19 pandemic on mental health in the general population: A systematic review. J Affect Disord 2020;277:55-64.

6. Wang C, Tee M, Roy Ae, et al. The impact of COVID-19 pandemic on physical and mental health of Asians: A study of seven middleincome countries in Asia. PLoS One 2021;16:e0246824.

7. Lee Y, Yang BX, Liu Q, et al. Synergistic effect of social media use and psychological distress on depression in China during the COVID-19 epidemic. Psychiatry Clin Neurosci 2020;74:552-4.

8. Low ZX, Yeo KA, Sharma VK, et al. Prevalence of Burnout in Medical and Surgical Residents: A Meta-Analysis. Int J Environ Res Public Health 2019;16:1479.

9. Chew NWS, Ngiam JN, Tan BY, et al. Asian-Pacific perspective on the psychological well-being of healthcare workers during the evolution of the COVID-19 pandemic. BJPsych Open 2020;6:e116.

10. Tan BYQ, Chew NWS, Lee GKH, et al. Psychological Impact of the COVID-19 Pandemic on Health Care Workers in Singapore. Ann Intern Med 2020;173:317-20.

11. Kirk AHP, Chong SL, Kam KQ, et al. Psychosocial impact of the COVID-19 pandemic on paediatric healthcare workers. Ann Acad Med Singap 2021;50:203-211.

12. Chew QH, Wei KC, Vasoo S, et al. Psychological and Coping Responses of Health Care Workers Toward Emerging Infectious Disease Outbreaks: A Rapid Review and Practical Implications for the COVID-19 Pandemic. J Clin Psychiatry 2020:81:20r13450.

13. Tng XJJ, Chew QH, Sim K. Psychological sequelae within different populations during the COVID-19 pandemic: a rapid review of extant evidence. Singapore Med J 2020. doi: 10.11622/ smedj.2020111.

14. Ho CS, Chee CY, Ho RC. Mental Health Strategies to Combat the Psychological Impact of COVID-19 Beyond Paranoia and Panic. Ann Acad Med Singap 2020;49:155-60. 